\title{
Atypical Hemolytic Uremic Syndrome in a One-Year Old Male Patient, Successfully Treated with Eculizumab
}

\section{Henri Fero ${ }^{1}$, Juna Musa ${ }^{2 *}$, Diamant Shtiza ${ }^{3}$, Ergys Cuka ${ }^{4}$, Loran Rakovica $^{5}$, Blina Abdullahu ${ }^{1}$, Klejda Hoxha ${ }^{1}$, Fareeha Nasir ${ }^{6}$ and Ali Guy}

${ }^{1}$ Medical Doctor, University of Hospital Center Mother Teresa, Tirane, Albania ${ }^{2}$ Postdoctoral Research Fellow, Department of Surgery, Mayo Clinic, Rochester, Minnesota, USA

${ }^{3}$ Professor Associate, Department Pediatrician Nephrology, University of Hospital Mother Teresa, Tirane, Albania

${ }^{4}$ Medical Doctor, University of Hospital Center Mother Teresa, Tirane, Albania

${ }^{5}$ Medical Doctor, University of Hospital in Prishtina, Kosovo

${ }^{6}$ Intern, Department of Surgery, Christian Hospital Quetta, Pakistan

${ }^{7}$ Department of Physical Medicine and Rehabilitation, School of Medicine - NUY

Medical Center, New York, USA

*Corresponding Author: Juna Musa, Postdoctoral Research Fellow, Department of Surgery, Mayo Clinic, Rochester, Minnesota, USA.
Received: April 12, 2020

Published: April 27, 2020

(C) All rights are reserved by Juna Musa., et al.

\begin{abstract}
Atypical hemolytic uremic syndrome is a rare form of thrombotic microangiopathies resulting from various genetic mutations of the complement factors. Thrombotic microangiopathies include Thrombotic thrombocytopenic purpura, primary HUS further divided into typical HUS or Shiga-toxin related HUS, atypical HUS and secondary forms of HUS.

The most common clinical features of the Hemolytic Uremic Syndrome are: kidney failure, thrombocytopenia resulting in hemorrhagic phenomena and also intravascular hemolytic anemia. Most commonly HUS is a diagnosis made from excluding all other plausible causes. HUS predominantly affects pediatric ages.
\end{abstract}

Keywords: Atypical Hemolytic Uremic Syndrome; Eculizumab; Thrombotic Microangiopathy

\section{Introduction}

Thrombotic Microangiopathy is a rare disorder that is characterized by thrombocytopenia, hemolytic anemia and thrombosis of the small arterioles that usually affects the kidneys and central nervous system. Evidence of the clinical and laboratory findings of intravascular hemolytic anemia combined with thrombocytopenia, renal failure and/or central nervous system, involvement in the absence of another plausible explanation is sufficient to diagnose TMA. What remains challenging to the physician is the differential diagnosis of TMA. First there is the differentiation of TTP and HUS. Even though they can overlap or coexist, we can confirm the presence of TTP by providing testing for the ADAMTS activity. Clinically TTP affects the CNS more often and causes less severe cases of kidney failure. In contrary HUS is clinically presented with severe kidney failure. Furthermore HUS is divided into secondary, due to infections such as HIV, drugs, pregnancy or autoimmune disease and primary which itself is divided into the classic HUS caused by the activity of E. Coli derived Shiga Toxin and atypical HUS or aHUS, a rare form of HUS that is strongly related to genetic mutations that affect the alternate complement pathway activity. Finally, aHUS remains a diagnosis of exclusion that if it is suspected several genetic tests can be performed but, they in fact, are not available in every clinic. We made a diagnose of exclusion to arrive at this diagnose.

\section{Case Presentation}

Here we present the case of a 1-year old male patient that was admitted to the pediatric intensive care unit with; a generalized severe condition, vomiting and diarrhea. The infant is agitated. Gross hematuria is noted. The skin and mucosa are pale. Periorbital and pitting edema in the lower extremities. BP of $140 / 90 \mathrm{~mm} / \mathrm{Hg}$.

Laboratory findings; anemia (Hb- 5,8 g/dl, RBC 2,223000/ $\mathrm{mm}^{3}$ ) thrombocytopenia $(97000 / \mathrm{mm} 3)$, WBC $\left(8000 / \mathrm{mm}^{3}\right)$, increased LDH and an increase in Urea and Creatinine $3.8 \mathrm{mg} / \mathrm{dL}$, Na $138 \mathrm{mEq} / \mathrm{L}, \mathrm{K} 5 \mathrm{mEq} / \mathrm{L}$, Cl 107mEq/L, and Metabolic Acidosis is noted with $\mathrm{HCO}_{3} 17.3 \mathrm{mEq} / \mathrm{L}$ Proteinuria and hemoglobinuria were noted in the urine analysis. Total bilirubin is elevated $(2 \mathrm{mg} /$ $\mathrm{dL})$, and direct bilirubin is normal $(0.2 \mathrm{mg} / \mathrm{dL})$. Prothrombin and aPTT time are within normal range.

Further examinations: Direct and Indirect COOMBS tests are negative, G-6-PD is within normal range, Hemoglobin electrophoresis is within normal range. Haptoglobin is low $20 \mathrm{mg} / \mathrm{dL}$ (41-165 $\mathrm{mg} / \mathrm{dL}$ ). Fibrinogen is low.

Blood microscopic examination revealed the presence of schistocytes. Reticulocyte count was high. Schistocytes on blood smear 
were the most important finding to conclude that the hemolytic anemia is intravascular.

The patient tested positive for CMV and Herpes Simplex virus.

Shiga toxin test and serology were negative. IgM anti-T antibody negative.

Low levels of C3 and C4 complement factors.

Further diagnostic tests to differentiate between thrombotic microangiopathy forms TTP/HUS were performed. The ADAMTS 13 activity test and ADAMTS 13 antibodies test was performed to rule out TTP and the results came back normal. Based on the clinical findings and laboratory studies, atypical Hemolytic Uremic Syndrome was suspected, in the setting of the HUS triad (intravascular hemolytic anemia, kidney failure and thrombocytopenia). TTP was excluded due to lack of neurologic involvement and due to the ADAMTS $13 \mathrm{Ab}$ test that resulted negative. All secondary causes of HUS were also excluded. Typical HUS was excluded due to the fact that Shiga Toxin test and serology were negative.

The patient was given diuretics, blood transfusions, fresh plasma, amlodipine and Eculizumab was started. Meningococcal vaccine was administrated within a week after Eculizumab was started. The patient condition ameliorated greatly within days after the initiation of therapy with Eculizumab.

\section{Discussion}

Atypical HUS is a multigenic complement-mediated disorder [1]. In many patients, a gastrointestinal or a urogenital infection precedes the clinical triad and leads to a HUS [2]. It is widely accepted that a HUS, is linked to genetically determined dysregulation of the alternative pathway of complement [3]. Complementmediated HUS is diagnosed clinically by the demonstration of complement dysregulation by antibodies against complement factors or mutations in complement protein genes. Genetic screening should include CD46, C3, THBD, CHFR1, CHFR5, CFH, CFI, CFB, and DGKE [4]. The annual incidence of aHUS in the US has been estimated to be of 1 - 2 cases/million inhabitants [5]. The onset of the disease usually occurs before the age of 18 (60\% vs. $40 \%)$ and sex characteristics are well-balanced (women are primarily affected when the disease is developed in adulthood) [6,7]. Diagnosis of the aHUS is based on excluding other causes. Hemolytic Uremic Syndrome should always be suspected based if there is evidence of the classic HUS triad (intravascular hemolytic anemia, kidney failure and thrombocytopenia). Differential diagnosis includes Thrombotic thrombocytopenic purpura (TTP) since both have different evolution and treatment but belong to the same group of histopathological characteristics known as Thrombotic Microangiopathies. TTP generally is known to have a higher prevalence of neurological involvement, ADAMTS 13 test positive for Anti-ADAMTS-13 autoantibody or low activity in secondary causes, multi-systemic organ damage and rarely, renal involvement. Prothrombin time and
aPTT within normal range is determinant to exclude Disseminated Intravascular Coagulation. Shiga-toxin related HUS should always be considered in case of HUS suspicion and ruled out if so in order to further investigate. aHUS, was suspected after negative results of stool examinations for Shiga Toxin and negative serology. Secondary causes of HUS (HIV, Neoplasia, autoimmune disorders) all should be carefully evaluated and ruled out.

First line therapy and drug of choice for aHUS treatment is Eculizumab. The efficacy and safety of eculizumab in inducing remission of acute episodes of aHUS and maintaining long-term remission, both in native kidneys and in the kidney grafts, was documented in two prospective clinical trials of primarily adult patients with plasma-dependent or plasma-resistant aHUS [8] and in a pediatric trial in 22 children, of whom 12 had no prior plasma treatment [9]. The main concern with eculizumab is increased susceptibility to gonococcal infections. For this reason, patients must receive meningococcal vaccination within 2 weeks. In addition, the risk for sensitization associated with chronic eculizumab exposure or with its deposition in tissue, and the recent report of hepatoxicity in pediatric patients [10] suggest that careful approach to withdrawal when-ever possible, should be attempted, under tight control of disease and complement activity [11]. Plasma therapy (Plasmapheresis or plasma infusion) has been a standard therapy for treatment of aHUS and should be started immediately associated with Eculizumab. Because $\mathrm{CFH}$ is a plasma protein, plasma infusion or exchange provides normal $\mathrm{CFH}$ to patients carrying $\mathrm{CFH}$ mutations $[12,13]$.

People with aHUS and ESRD have also had to undergo lifelong dialysis, which has a 5 -year survival rate of $34-38 \%[14,15]$.

\section{Conclusion}

aHUS, is a very challenging diagnosis for every physician as it includes multiple differential diagnoses and also a variety of examinations that include genetic testing. Treatment with Eculizumab is showing promising results and many patients have better prognoses than patients who only receive plasma therapy as in our case. However, Eculizumab does not treat the underlying cause of the disease. It is now being proposed that a definite treatment for patients who have a poor prognosis and those who have end stage renal disease, a hepato-renal transplant is seen as a solution in order to not only restore the renal function, but also to produce non mutant complement factors.

\section{Bibliography}

1. Noris M and Remuzzi G. "Hemolytic uremic syndrome". Journal of the American Society of Nephrology 16.4 (2005): 1035.

2. Nester CM., et al. "Atypical aHUS: state of the art". Molecular Immunology 67.1 (2015): 31-42.

3. Noris M and Remuzzi G. "Atypical hemolytic-uremic syndrome". New England Journal of Medicine 361.17 (2009): 1676-1687. 
4. Goodship TH., et al. "Atypical hemolytic uremic syndrome and C3 glomerulopathy: conclusions from a Kidney". Kidney International 91.3 (2017): 539-551.

5. AR Constantinescu., et al. "Non-enteropathic hemolytic uremic syndrome: causes and short-term course". American Journal of Kidney Diseases 43.6 (2004): 976-982.

6. AL Sellier-Leclerc., et al. "Differential impact of complement mutations on clinical characteristics in atypical hemolytic uremic syndrome". Journal of the American Society of Nephrology 18.8 (2007): 2392-2400.

7. M Sullivan., et al. "Epidemiological approach to identifying genetic predispositions for atypical hemolytic uremic syndrome". Annals of Human Genetics 74.1 (2010): 17-26.

8. Legendre CM., et al. "Terminal complement inhibitor eculizumab in atypical hemolytic-uremic syndrome". New England Journal of Medicine 368.23 (2013): 2169-2181.

9. Greenbaum LA., et al. "Eculizumab is a safe and effective treatment in pediatric patients with atypical hemolytic uremic syndrome". Kidney International 89.3 (2016): 701-711.

10. Hayes W., et al. "Eculizumab hepatotoxicity in pediatric aHUS". Pediatric Nephrology 30.5 (2015): 775-781.

11. Noris M., et al. "Dynamics of complement activation in aHUS and how to monitor eculizumab therapy". Blood 124.11 (2014): 1715-1726.

12. Caprioli J., et al. "Genetics of HUS: the impact of MCP, CFH, and IF mutations on clinical presentation, response to treatment, and outcome". Blood 108.4 (2006): 1267-1279.

13. Noris M., et al. "Relative role of genetic complement abnormalities in sporadic and familial aHUS and their impact on clinical phenotype". Clinical Journal of the American Society of Nephrology 5.10 (2010): 1844-1859.

14. Collins Allan J., et al. "Excerpts from the US Renal Data System 2009 Annual Data Report”. American Journal of Kidney Diseases 55.1 (2010): S1-S7.

15. European Renal Association- European Dialysis and Transplant Association Registry. "ERA-EDTA Registry Annual Report 2009". Amsterdam, The Netherlands: Academic Medical Center Department of Medical Informatics (2011).

\section{Assets from publication with us}

- Prompt Acknowledgement after receiving the article

- Thorough Double blinded peer review

- Rapid Publication

- Issue of Publication Certificate

- High visibility of your Published work

Website: https://www.actascientific.com/

Submit Article: https://www.actascientific.com/submission.php

Email us: editor@actascientific.com

Contact us: +919182824667 